\title{
Pollen Preservation Potential of Imperata cylindrica - an Efficient Source for Doubled Haploid Production in Wheat
}

\author{
S.A. RATHER* ${ }^{*}$ H.K. Chaudhary and V. KaIla \\ Molecular Cytogentics \& Tissue Culture Lab, Department of Crop Improvement, \\ CSK HP Agricultural University, Palampur-176062, Himachal Pradesh, India
}

(Received 22 August 2016; Accepted 23 January 2017;

Communicated by I. Molnár)

\begin{abstract}
Pollen of two I. cylindrica genotypes, one having spike with brown anthers (Ic-Pbr) and other with yellow anthers (Ic-Pye) was collected from three collection environments (early, mid and late flowering) and preserved in sealed Petri dishes under three preservation regimes, P1 $\left(4{ }^{\circ} \mathrm{C}\right.$ temperature and $\left.60 \% \mathrm{RH}\right), \mathrm{P} 2\left(-20{ }^{\circ} \mathrm{C}\right.$ and $\left.65 \% \mathrm{RH}\right)$ and $\mathrm{P} 3\left(-80{ }^{\circ} \mathrm{C}\right.$ temperature and $45 \% \mathrm{RH})$. The pollen viability and germination tests of the preserved pollen were conducted after every 15 days till complete loss of viability. The study revealed that Ic-Pbr was superior to Ic-Pye in terms of the mean absolute pollen viability (APV) under preservation conditions. Among the collection environments, pollen collected just after first anthesis, that is, early flowering was superior in terms of the preservation potential. The preservation regime, $\mathrm{P} 2$ was found to be best among the three for better preservation of I. cylindrica pollen. By utilizing the one month old preserved pollen for pollination of wheat spikes, pollen from $\mathrm{P} 2$ regime induced haploid embryos to the tune of $25.53 \%$. The study concluded that by preserving I. cylindrica pollen at $-20{ }^{\circ} \mathrm{C}$, the period of $\mathrm{DH}$ production in wheat can be extended by at least one month. The preserved pollen will also open avenues for exploring the possibilities of inducing haploids in other cereals like rice and maize whose flowering does not coincide with I. cylindrica naturally.
\end{abstract}

Keywords: pollen, preservation regimes, absolute pollen viability, haploid

\section{Introduction}

Doubled haploidy breeding serves as a useful tool in wheat improvement programmes by providing a means of achieving instant homozygosity, of desirable characters of the recombinants. Of the various techniques for haploid production in wheat (Triticum aestivum L.), chromosome elimination-mediated approach has been studied and investigated by researchers to a great extent and nowadays is being utilized for large scale practical applications. Lately, wheat $\times$ Imperata cylindrica-mediated chromosome elimination approach of haploid induction has taken advantage over all the existing systems and techniques in enhancing the efficiency of developing complete homozygous lines in wheat (Chaudhary et al. 2005a). There is scope of exploring the possibilities of utilizing this system for developing haploids in other important cereals like rice and maize and also for

*Corresponding author; E-mail: shoukat.pbg@gmail.com; Mobile: +91-7464825790 
extending the period of developing haploids in late flowering, late sown and diploid progenitors of bread wheat whose flowering does not coincide with I. cylindrica under natural conditions.

Pollen preservation is one such approach that allows crossing of plants whose flowering does not coincide or which may be growing in distant parts of the world (Towill 2004). The preserved pollen can be used for extending the duration of doubled haploidy breeding programmes in wheat (Inagaki and Mujeeb-Kazi 1994, 1998). Moreover, it may eliminate the requirement of synchronizing the flowering of the female and the pollen parent and will allow wide hybridization across seasonal and geographical limitations (Bajaj 1987). The stored pollen can also be shipped internationally, often without any disease transfer (Hoekstra 1995).

Preservation of pollen of any plant species will only be useful if viability and germination of preserved pollen is maintained. Pollen longevity among different species varies from minutes to years depending primarily on the taxa of the plant and on the abiotic environmental conditions. Although, pollen viability studies in respect of I. cylindrica under storage conditions have not been reported, yet various other members of the family Gramineae are investigated by different researchers for this trait (Stanley and Linskens 1974; Barnabas and Rajki 1981; Hanna and Towill 1995). Besides the use of preserved pollen in hybridization programmes, it may be used for the study of the pollen biology, genetics, pollen-stigma interaction, incompatibility and fertility studies (Stanley and Linskens 1974; Heslop-Harrison et al. 1984; Heslop-Harrison and Heslop-Harrison 1992; Mulugeta et al. 1994).

Keeping in view the above, the present study was conducted to find out the best preservation regime and the time for which the pollen of I. cylindrica can be preserved in a viable state, so that, the period of doubled haploid production in wheat can be extended by pollinating late sown, late flowering and greenhouse grown (off season) wheat varieties with the preserved pollen. Moreover, the preserved I. cylindrica pollen can be used for studies on exploration of possibilities of developing haploids in other important cereals like rice, maize and diploid progenitors of bread wheat whose flowering does not coincide with I. cylindrica naturally.

\section{Materials and Methods}

To undertake the present investigation, pollen of two local and wildly growing diverse I. cylindrica genotypes (spike with brown anthers (Ic-Pbr) and spike with yellow anthers (Ic-Pye)) (Figs S1 and S2*) was freshly collected during morning hours (7 AM) during its flowering period (April month) in small Petri dishes and sealed instantly with parafilm. Three pollen collections, classified as collection environments (E1, E2 and E3) of each genotype were made on different dates $5^{\text {th }}$ April, $20^{\text {th }}$ April and $5^{\text {th }}$ May, 2011, respectively at 15 days interval to investigate the role of atmospheric temperature and humidity at the time of pollen harvest on pollen longevity under preserved conditions. The atmos-

\footnotetext{
*Further details about the Electronic Supplementary Material (ESM) can be found at the end of the article.
} 
pheric temperature and relative humidity (\%) on different days of pollen collection are given in Table S1. The collected pollen in sealed Petri dishes was stored under varied preservation regimes, viz. P1 $\left(4{ }^{\circ} \mathrm{C}\right.$ temperature and $\left.60 \% \mathrm{RH}\right), \mathrm{P} 2\left(-20{ }^{\circ} \mathrm{C}\right.$ temperature and $65 \% \mathrm{RH})$ and $\mathrm{P} 3\left(-80{ }^{\circ} \mathrm{C}\right.$ temperature and $\left.45 \% \mathrm{RH}\right)$.

On each day of collection, 73 Petri dishes of each genotype were collected, out of which one Petri dish was instantly used for checking viability and germination of fresh pollen on the same day of collection (control), whereas 24 Petri dishes, out of the rest, were preserved at each preservation regime for carrying out viability and germination tests after every 15 days till the pollen lost its viability completely. The 73 Petri dishes were collected so that viability tests can be conducted throughout the year till next wheat crop as two Petri dishes were used for viability test each month. The similar procedure of pollen collection was followed for three different dates.

The accuracy of different protocols for assessment of pollen viability and germination was determined by observing the staining and germination of the fresh and pollen killed at $80{ }^{\circ} \mathrm{C}$ for 24 hours and the protocols given under were found most appropriate. The correlation of the results obtained from in vitro pollen viability and germination tests was determined by pollinating the Saptdhara variety of bread wheat by one month old pollen preserved under different preservation regimes.

\section{Pollen viability test}

The pollen grains were stained in the mixture of $1 \%$ acetocarmine (Himedia) and glycerine (Himedia) in the ratio of 1:1 for 1 to 2 hours and observed under bright field microscope (Olympus BX 61). The fully stained pollen grains were counted as viable (Singh

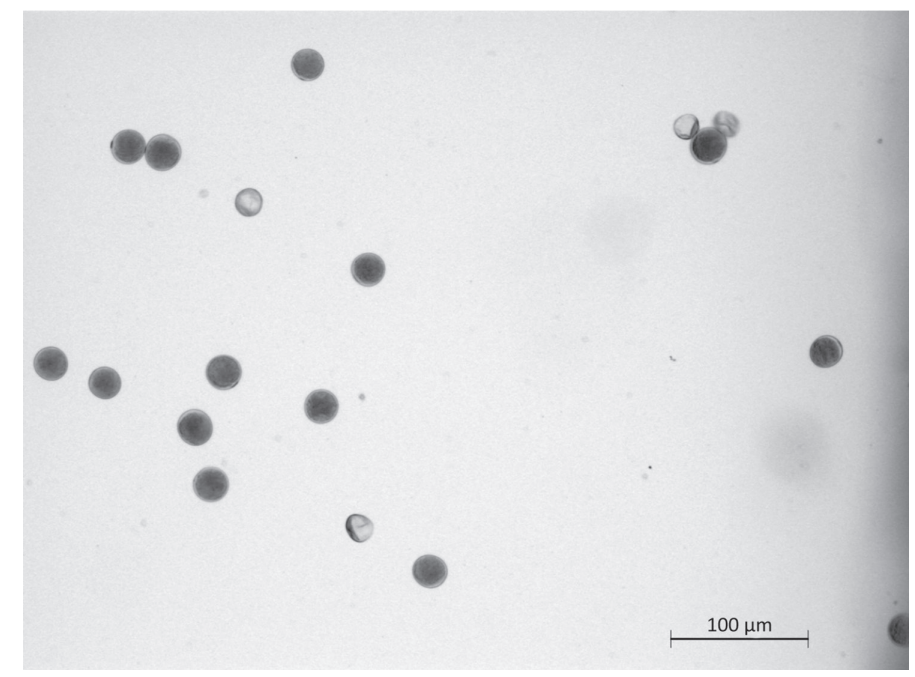

Figure 1. Darkly stained viable and unstained non-viable Imperata cylindrica pollen grains upon treatment with acetocarmine 
2003; Chaudhary et al. 2005a). The viable and non-viable pollen grains of I. cylindrica are shown in Fig. 1.

\section{Pollen germination test}

The protocol given by Kubo (1958) was used for assessing the germination of I. cylindrica pollen with slight modifications. The pollen germination medium comprised of $4 \%$ sucrose (Himedia) and $0.5 \mathrm{~g}$ agar-agar (Himedia), dissolved and boiled in $25 \mathrm{ml}$ distilled water. After cooling the boiling mixture to $35^{\circ} \mathrm{C}, 0.5 \mathrm{~g}$ gelatin (Himedia) was added and the mixture was stirred until glycerin melts. A thin film of the solution was smeared on a glass slide with another glass slide and then the pollen grains were dusted on the slide with a camel brush. The cover slip placed on the slide was sealed with glycerine from the sides. The slides were then kept in a moist chamber for germination in an incubator at $35^{\circ} \mathrm{C}$ and the progress of the germination was observed frequently under phase contrast microscope (Olympus CX 31). The pollen grains whose pollen tube length was at least equal to or more than their diameter after 24 hours were counted as germinated (Chaudhary et al. 2005a). The germinated and non-germinated pollen grains of I. cylindrica are shown in Fig. 2.

In each viability and germination test of the preserved pollen from each preservation regime, five slides with 10 random samples (each sample corresponding to a microscopic field with 50-60 pollen grains) in each slide were observed under the microscope for working out the pollen viability and germination percentage on different dates. After working out the pollen viability and germination percentage as average of various observations, absolute pollen viability (APV) was worked out as per the formula used by Visser et al. (1977) as:

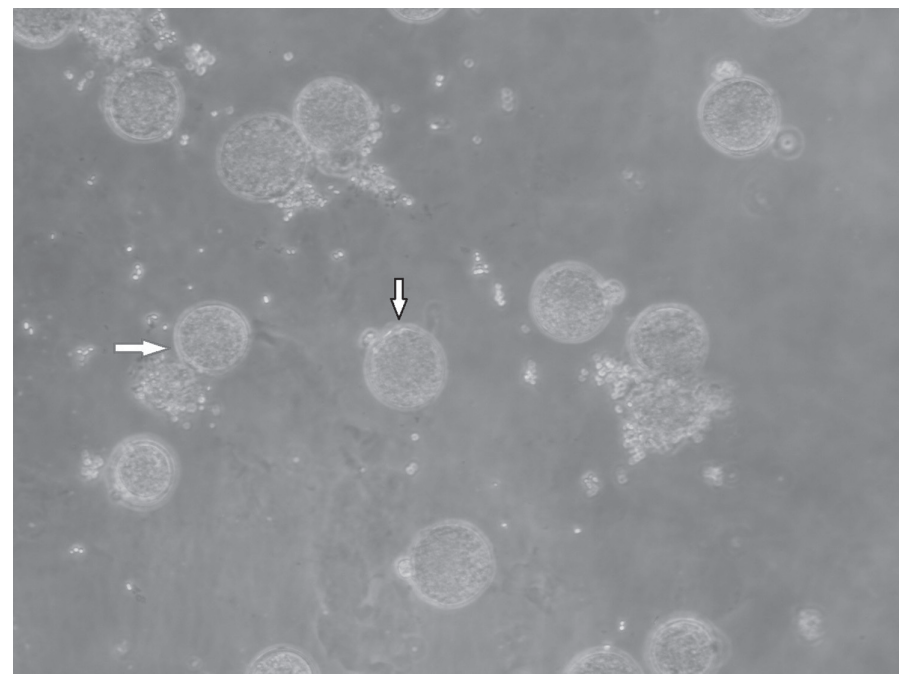

Figure 2. Germinating (red arrow) and non-germinating (black arrow) Imperata cylindrica pollen grains on germination medium 


$$
\mathrm{APV}=\frac{\% \text { Viability } \times \% \text { Germination }}{100}
$$

The data obtained on absolute viability ( $\%$ ) of the pollen of two I. cylindrica genotypes collected from different collection environments and preserved under varied preservation regimes was analyzed as per factorial randomized complete block design separately for each in vitro test (Rangaswamy 2006). The critical difference (CD) for comparison within genotypes, collection environments and preservation regimes was calculated as:

$$
\mathrm{CD}=\text { S.E. }(\mathrm{d}) \times \text { ' } \mathrm{t} \text { ' value (at error } \mathrm{df} \text { and } 0.05 \text { level of significance) }
$$

where, S.E. (d) for $\mathrm{X}= \pm(2 \mathrm{EMS} / \mathrm{rD})^{1 / 2}$, in which $\mathrm{X}=$ the main factor or interaction, $\mathrm{D}=$ the product of the levels of the left out factors and $\mathrm{r}=$ number of replications.

\section{Results}

On the day of collection ( 0 days), both the collection environments and $I$. cylindrica genotypes showed significant differences for absolute pollen viability (\%). The different collection environments during which the pollen of $I$. cylindrica genotypes was collected possessed significant differences for absolute viability in all the in vitro tests as revealed by analysis of variance, whereas, the preservation regimes exhibited significant differences for absolute viability at 15, 30, 45, 60, 75, 90, 105, 120 and 135 days after collection. Likewise, the genotypic differences existed only at 15, 90, 105, 120, 150, 165, 180 and 255 days after collection. The interactions including $\mathrm{P} \times \mathrm{E}, \mathrm{G} \times \mathrm{E}, \mathrm{G} \times \mathrm{P}$ and $\mathrm{G} \times \mathrm{P} \times \mathrm{E}$ also exhibited significant differences for absolute pollen viability (APV) at different days after pollen collection (ANOVA tables not given).

The I. cylindrica genotypes, Ic-Pbr and Ic-Pye exhibited significant differences from mean for APV averaged over replications, collection environments and preservation regimes at 15, 90, 105, 120, 150, 165, 180 and 255 days after collection (Table 1). The mean absolute viability of freshly collected pollen of genotype Ic-Pbr $(64.97 \%)$ was significantly higher than that of genotype Ic-Pye (62.69\%). After one fortnight of preservation, mean APV of the genotype Ic-Pbr (48.68\%) was significantly higher as compared to that of the genotype Ic-Pye (44.90\%). Likewise, genotype Ic-Pbr was found significantly superior to genotype Ic-Pye for the mean APV worked out after sixth, seventh, eighth, $10^{\text {th }}$, $11^{\text {th }}, 12^{\text {th }}$ and $17^{\text {th }}$ fortnight of preservation (Table 2).

The collection environments E1, E2 and E3 possessed significant differences from mean for APV averaged over replications, genotypes and preservation regimes throughout the preservation duration (Table 3 ). The mean absolute viability of freshly collected pollen from collection environment E1 (67.87\%) was significantly higher than that of collection environments E2 (62.73\%) and E3 (60.90\%). After one fortnight of preservation, the mean absolute viability of pollen collected from collection environment E1 $(48.93 \%)$ was significantly superior to that of collection environments E2 $(47.30 \%)$ and E3 (44.12\%). Similarly, after one month of preservation, the mean absolute viability of 


\begin{tabular}{|c|c|c|c|c|}
\hline 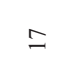 & $\stackrel{*}{\stackrel{*}{*}}$ & ñ & : & $\stackrel{t}{0}$ \\
\hline$\stackrel{\bullet}{\sim}$ & $\stackrel{n}{-}$ & $\stackrel{\infty}{n}$ & $\stackrel{\sigma}{-}$ & $\stackrel{0}{0}$ \\
\hline$\simeq$ & 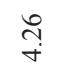 & $\hat{\sigma}$ & $\stackrel{\circ}{\underset{f}{f}}$ & $\stackrel{2}{\stackrel{5}{0}}$ \\
\hline \pm & مे & $\stackrel{\infty}{\sim}$ & $\underset{⿱}{\frac{\pi}{2}}$ & $\hat{n}$ \\
\hline 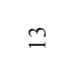 & $\stackrel{2}{\Xi}$ & $\stackrel{\infty}{\infty}$ & $\stackrel{\text { I্ }}{=}$ & $\tilde{n}$ \\
\hline$\simeq$ & $\stackrel{*}{\stackrel{*}{\sim}}$ & ڤ̆ & $\stackrel{n}{m}$ & $\stackrel{?}{+}$ \\
\hline$=$ & $\begin{array}{l}* \\
\infty \\
\infty \\
\end{array}$ & 。․ & 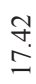 & กี \\
\hline$\stackrel{\circ}{\circ}$ & 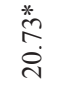 & $\begin{array}{l}0 \\
\stackrel{2}{2}\end{array}$ & $\begin{array}{l}\text { ¿े. } \\
\stackrel{\text { ¿े }}{ }\end{array}$ & กิ \\
\hline$a$ & 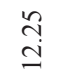 & 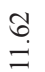 & $\stackrel{ \pm}{=}$ & $\stackrel{0}{+}$ \\
\hline$\infty$ & $\begin{array}{l}\text { 今ે } \\
\stackrel{\text { ले }}{=}\end{array}$ & $\begin{array}{l}\hat{b} \\
\infty\end{array}$ & $\stackrel{\overbrace{}}{\circ}$ & ते \\
\hline$r$ & $\begin{array}{l}\stackrel{*}{\circ} \\
\stackrel{\sigma}{ٍ}\end{array}$ & $\begin{array}{l}\stackrel{0}{=} \\
=\end{array}$ & $\begin{array}{l}\stackrel{n}{n} \\
\text { co }\end{array}$ & ñ? \\
\hline 6 & $\begin{array}{l}* \\
6 \\
6 \\
0\end{array}$ & $\begin{array}{l}\vec{\infty} \\
\ddot{n}\end{array}$ & సֶ. & $\stackrel{9}{\mathfrak{0}}$ \\
\hline in & $\begin{array}{l}\stackrel{+}{\sim} \\
\stackrel{N}{0}\end{array}$ & $\begin{array}{l}\infty \\
\infty \\
\stackrel{\infty}{\sim}\end{array}$ & $\begin{array}{l}\stackrel{\circ}{\circ} \\
\stackrel{\sim}{*}\end{array}$ & $\hat{n}$ \\
\hline$\sigma$ & 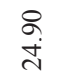 & $\stackrel{\grave{N}}{\stackrel{\sim}{\sim}}$ & $\begin{array}{l}\stackrel{\infty}{n} \\
\stackrel{\sim}{\sim}\end{array}$ & $\stackrel{9}{0}$ \\
\hline$m$ & $\stackrel{\text { f̊ }}{\stackrel{0}{0}}$ & $\begin{array}{l}\infty \\
\stackrel{\lambda}{\hat{\lambda}}\end{array}$ & $\begin{array}{l}\text { ते } \\
\text { ळे }\end{array}$ & $\stackrel{0}{\stackrel{0}{0}}$ \\
\hline$N$ & $\underset{\infty}{\infty}$ & $\underset{m}{\stackrel{m}{m}}$ & 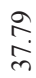 & $n$ \\
\hline- & $\begin{array}{l}* \\
\infty \\
0 \\
\infty \\
\dot{\infty}\end{array}$ & $\begin{array}{l}\stackrel{8}{\circ} \\
\dot{7}\end{array}$ & $\begin{array}{l}2 \\
6 \\
0\end{array}$ & $\stackrel{+}{n}$ \\
\hline 0 & 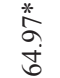 & $\begin{array}{l}\text { oे } \\
\text { రु }\end{array}$ & $\begin{array}{l}\tilde{\infty} \\
\tilde{\sigma}\end{array}$ & $\stackrel{0}{0}$ \\
\hline $\begin{array}{l}\vec{s}^{*} \\
\stackrel{*}{*} \\
\stackrel{*}{*}\end{array}$ & 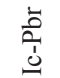 & 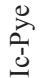 & 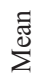 & Oి ì \\
\hline & \multicolumn{2}{|c|}{$\begin{array}{c}(\%) \\
\Lambda \mathrm{dV} \text { urəəW }\end{array}$} & & \\
\hline
\end{tabular}

\begin{tabular}{|c|c|c|c|c|c|}
\hline$=$ & 葛 & 1 & ñ & 3 & $\stackrel{t}{0}$ \\
\hline$\simeq$ & $\stackrel{*}{\stackrel{*}{+}}$ & ț & 동 & $\stackrel{\vec{\sigma}}{-}$ & $\frac{a}{0}$ \\
\hline 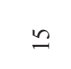 & $\stackrel{*}{*}$ & $\underset{\sim}{\sim}$ & $\underset{r}{\stackrel{J}{J}}$ & $\underset{+}{\stackrel{f}{+}}$ & $\ddot{n}$ \\
\hline \pm & 菑 & $\hat{\sigma}$ & f? & $\underset{r}{\stackrel{8}{r}}$ & $\stackrel{?}{0}$ \\
\hline 2 & $\begin{array}{l}{ }_{0}^{*} \\
\infty \\
i \\
i\end{array}$ & $\hat{a}$ & $\stackrel{\circ}{\circ}$ & $\stackrel{\text { I্ }}{=}$ & $\stackrel{n}{?}$ \\
\hline$\simeq$ & $\begin{array}{l}* \\
\infty \\
\infty \\
i\end{array}$ & $\stackrel{\infty}{\stackrel{n}{ \pm}}$ & $\begin{array}{l}\underset{J}{J} \\
\text { I }\end{array}$ & $\stackrel{\tilde{m}}{ \pm}$ & 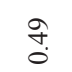 \\
\hline$=$ & $\begin{array}{l}* \\
\stackrel{*}{\infty} \\
\infty\end{array}$ & 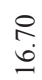 & $\begin{array}{l}8 \\
\stackrel{0}{0}\end{array}$ & $\stackrel{\overbrace{}}{\stackrel{f}{\leftrightarrows}}$ & +̊. \\
\hline 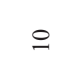 & \begin{tabular}{l}
$*$ \\
\multirow{2}{0}{} \\
$i$ \\
\end{tabular} & $\stackrel{\text { f }}{2}$ & $\begin{array}{l}0 \\
\stackrel{0}{0}\end{array}$ & $\begin{array}{l}\text { ¿े } \\
\stackrel{\text { ते }}{ }\end{array}$ & 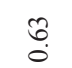 \\
\hline$a$ & $\begin{array}{l}* \begin{array}{c}* \\
n \\
n \\
n\end{array}\end{array}$ & $\stackrel{\stackrel{n}{=}}{=}$ & $\stackrel{\overrightarrow{0}}{\stackrel{0}{0}}$ & $\stackrel{+}{=}$ & $\begin{array}{l}0 \\
n \\
0\end{array}$ \\
\hline$\infty$ & $\stackrel{*}{\stackrel{*}{?}} \stackrel{\text { ב }}{=}$ & 훙 & $\begin{array}{l}+ \\
\infty \\
\infty\end{array}$ & $\stackrel{\overbrace{}}{\circ}$ & $\stackrel{n}{n}$ \\
\hline r & 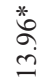 & $\stackrel{\infty}{\stackrel{\infty}{=}}$ & $\stackrel{\underset{+}{ \pm}}{=}$ & 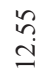 & $\stackrel{n}{\stackrel{n}{0}}$ \\
\hline 0 & $\begin{array}{l}* \\
\infty \\
\infty \\
\infty \\
\infty\end{array}$ & $\begin{array}{l}\stackrel{ }{\infty} \\
\dot{I}\end{array}$ & $\begin{array}{l}\overrightarrow{7} \\
\stackrel{\imath}{\imath}\end{array}$ & సู & n̊. \\
\hline in & $\frac{*}{a}$ & $\begin{array}{l}\infty \\
\substack{\infty \\
\infty}\end{array}$ & $\begin{array}{l}\infty \\
\infty \\
\stackrel{\infty}{-}\end{array}$ & $\begin{array}{l}\stackrel{\sim}{\circ} \\
\stackrel{\sim}{0}\end{array}$ & $\stackrel{n}{\circ}$ \\
\hline t & \begin{tabular}{l}
$*$ \\
\multirow{2}{0}{} \\
0 \\
0
\end{tabular} & $\begin{array}{l}\infty \\
\text { ते } \\
\text { N }\end{array}$ & $\begin{array}{l}\triangleright \\
\dot{\sim} \\
\dot{\sim}\end{array}$ & 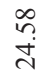 & $\stackrel{8}{8}$ \\
\hline m & $\begin{array}{l}* \\
\tilde{n} \\
\sim\end{array}$ & $\begin{array}{l}\tilde{d} \\
\infty \\
\text { तो }\end{array}$ & $\begin{array}{l}\infty \\
\stackrel{2}{2} \\
\grave{2}\end{array}$ & ָ̊ & $\begin{array}{l}0 \\
? \\
0\end{array}$ \\
\hline N & 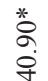 & 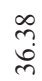 & $\begin{array}{l}\infty \\
\stackrel{0}{0} \\
\dot{\Gamma}\end{array}$ & $\underset{i}{\stackrel{2}{n}}$ & $\hat{0}$ \\
\hline- & $\stackrel{*}{*}$ & 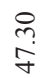 & $\stackrel{\stackrel{ }{7}}{\underset{f}{f}}$ & $\stackrel{\overbrace{}}{\hat{b}}$ & : \\
\hline 0 & 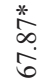 & $\stackrel{\overbrace{}}{\stackrel{\Omega}{\delta}}$ & $\begin{array}{l}\stackrel{8}{8} \\
8\end{array}$ & $\begin{array}{l}\tilde{\infty} \\
\tilde{6}\end{array}$ & $\stackrel{\overbrace{}}{\sim}$ \\
\hline $\begin{array}{l}\vec{e}_{\hat{e}}^{*} \\
\stackrel{*}{*}\end{array}$ & 弐 & 포 & ભ & $\sum_{\Sigma}^{\bar{\Xi}}$ & 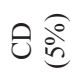 \\
\hline & \multicolumn{3}{|c|}{ (\%) $\Lambda \mathrm{dV}$ uвәW } & & \\
\hline
\end{tabular}


pollen collected from collection environment E1 (40.90\%) was significantly superior to that of collection environments E2 (36.38\%) and E3 (36.08\%) and parallel trend was followed throughout the preservation duration (Table 2).

The preservation regimes P1, P2 and P3 exhibited significant differences from mean for average APV throughout the preservation duration. The mean absolute viability of pollen preserved under preservation regime P2 $(54.96 \%)$ was significantly higher than that of preservation regimes $\mathrm{P} 1$ (46.45\%) and P3 (38.94\%) after one fortnight of preservation. Likewise, after one month of preservation duration, the mean absolute viability of pollen in preservation regime P2 (46.97\%) was statistically higher than that of preservation regimes $\mathrm{P} 1(37.74 \%)$ and $\mathrm{P} 3(28.65 \%)$ and corresponding trend was followed throughout the preservation duration (Table 3 ).

The in vivo test of preserved pollen was also carried out by pollinating bread wheat with one month old pollen preserved under varied preservation regimes. Upon pollination of 'Saptdhara' variety of bread wheat with one month old pollen preserved under different preservation regimes $\mathrm{P} 1, \mathrm{P} 2$ and $\mathrm{P}$, the haploid embryo formation frequency obtained

Table 3. Mean absolute viability over replications, collection environments and genotypes of pollen preserved under varied preservation regimes (P1, P2 and $\mathrm{P} 3)$

\begin{tabular}{|c|c|c|c|c|c|c|c|c|c|c|}
\hline & $\begin{array}{l}\text { Test } \\
\text { No.** }\end{array}$ & 1 & 2 & 3 & 4 & 5 & 6 & 7 & 8 & 9 \\
\hline \multirow{3}{*}{ 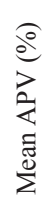 } & P1 & 46.45 & 37.74 & 28.64 & 22.44 & 17.37 & 11.31 & 6.7 & 3.53 & 1.24 \\
\hline & P2 & $54.96^{*}$ & $46.97 *$ & $40.50^{*}$ & $37.05^{*}$ & $33.79 *$ & $32.06 *$ & $29.26^{*}$ & $25.85^{*}$ & $22.64 *$ \\
\hline & P3 & 38.94 & 28.65 & 21.46 & 14.26 & 9.01 & 5.28 & 1.69 & 0.71 & 38.94 \\
\hline & Mean & 46.79 & 37.79 & 30.2 & 24.58 & 20.06 & 16.22 & 12.55 & 10.03 & 11.94 \\
\hline & $\begin{array}{c}\text { CD } \\
(5 \%)\end{array}$ & 0.66 & 0.67 & 0.56 & 0.60 & 0.45 & 0.50 & 0.45 & 0.35 & 0.46 \\
\hline
\end{tabular}

$* \mathrm{P} \leq 0.05$. **Number of fortnights after the collection of pollen.

Table 4. Validation of in vitro absolute pollen viability results and comparison of preservation regimes (P1, P2 and P3) by in vivo test of one month old preserved pollen

\begin{tabular}{|c|c|c|c|c|c|c|c|c|c|c|c|c|}
\hline \multirow{3}{*}{ Genotype } & \multicolumn{3}{|c|}{ Fresh pollen } & \multicolumn{6}{c|}{ One month old pollen preserved under } \\
\cline { 2 - 13 } & \multicolumn{3}{|c|}{ Control } & \multicolumn{3}{c|}{ P1 } & \multicolumn{3}{c|}{ P2 } & \multicolumn{3}{c|}{ P3 } \\
\cline { 2 - 12 } & f & sf & ef & f & sf & ef & f & sf & ef & f & sf & Ef \\
\hline Saptdhara & 85 & $\begin{array}{c}69.41 \\
(59)\end{array}$ & $\begin{array}{c}32.20 \\
(19)\end{array}$ & 81 & $\begin{array}{c}37.04 \\
(30)\end{array}$ & $\begin{array}{c}13.33 \\
(4)\end{array}$ & 89 & $\begin{array}{c}52.81 \\
(47)\end{array}$ & $\begin{array}{c}25.53 \\
(12)\end{array}$ & 72 & $\begin{array}{c}26.39 \\
(19)\end{array}$ & $\begin{array}{c}10.53 \\
(2)\end{array}$ \\
\hline
\end{tabular}

$\mathrm{f}=$ total number of florets pollinated; $\mathrm{sf}=$ pseudoseed formed; ef $=$ embryo formation.

Figures in parenthesis represent the number obtained; $\mathrm{P} 1=4{ }^{\circ} \mathrm{C} \& 60 \% \mathrm{RH} ; \mathrm{P} 2=-20{ }^{\circ} \mathrm{C} \& 65 \% \mathrm{RH} ; \mathrm{P} 3=-80{ }^{\circ} \mathrm{C} \& 45 \% \mathrm{RH}$ 
was $13.33 \%, 25.53 \%$ and $10.53 \%$, respectively. Whereas, the haploid embryo formation frequency obtained by fresh pollen was found to be $32.20 \%$ (Table 4 ).

In case of preservation regime $\mathrm{P} 1$, the absolute viability of the pollen of both the genotypes collected from all the three collection environments reduced to minimum after 135 days of preservation. In case of preservation regime P2, the APV of both the genotypes collected from collection environments E1 and E3 decreased to minimum after 255 days of preservation whereas, it happened so after 240 days of preservation in case of collection environment E2. In case of preservation regime P3, the APV of both the genotypes collected from all the three collection environments reduced to minimum after 120 days of preservation.

\section{Discussion}

Preservation of pollen of any plant species facilitates and allows wide hybridization across seasonal and geographical limitations (Bajaj 1987). The preserved pollen of pearl millet and maize has been successfully used for extending the doubled haploidy breeding programmes in wheat. Although, wheat $\times$ Imperata cylindrica has proved to be very efficient for $\mathrm{DH}$ production in wheat, still there is scope of further enhancing the efficiency of the system through preservation of I. cylindrica pollen. The preserved pollen will facilitate the extension of $\mathrm{DH}$ breeding programmes in wheat. At the same time, the preserved pollen of I. cylindrica can also be used for undertaking DH breeding programmes in wheat at places where the wild grass, I. cylindrica is not growing naturally.

In the present investigation, the analysis of variance revealed significant differences among I. cylindrica genotypes, one having spike with brown anthers and other with yellow anthers for absolute viability of the fresh pollen collected from different collection environments. The analysis of variance also revealed significant differences for absolute viability of pollen collected from different collection environments and preserved under varied preservation regimes throughout the preservation duration whereas, the genotypes and interactions among main effects (collection environments, preservation regimes and genotypes) exhibited significant differences in some tests only.

The genotype Ic-Pbr had higher absolute pollen viability (\%) as compared to the other genotype Ic-Pye, especially up to 15 days of preservation. Keeping in view the experimental yard stick for pollen viability laid by Kumar et al. (1995), absolute pollen viability of the genotype Ic-Pbr (slightly below 50\%) was significantly superior to that of IcPye at 15 days of preservation.

According to Towill (2004) and Ganeshan et al. (2008), the pollen to be collected for preservation should be harvested soon after anthesis. The results of the present investigation also revealed that the I. cylindrica pollen collected during the initial days of its flowering in the month of April had higher absolute viability than that collected during mid or end of its flowering. From these results, it can be conferred that for preservation, the I. cylindrica pollen must be collected during the initial days of its flowering, so that the preserved pollen can be efficiently used for extending or undertaking DH breeding programmes across seasonal or geographical limitations. 
Weatherhead et al. (1978) reported that potato pollen showed a decrease in germination to less than $1 \%$ of that of fresh pollen after 9 months of storage at $-20{ }^{\circ} \mathrm{C}$. The results obtained in the present investigation also revealed that the preservation regime $\mathrm{P} 2\left(-20^{\circ} \mathrm{C}\right.$ and $65 \% \mathrm{RH})$ was better than preservation regimes $\mathrm{P} 1\left(4^{\circ} \mathrm{C}\right.$ and $\left.60 \% \mathrm{RH}\right)$ and $\mathrm{P} 3\left(-80{ }^{\circ} \mathrm{C}\right.$ and $45 \% \mathrm{RH}$ ), both in terms of duration for which the pollen can be preserved as well as in vitro absolute pollen viability throughout the preservation duration. The in vivo test also revealed that the one month old pollen preserved at $-20{ }^{\circ} \mathrm{C}(65 \% \mathrm{RH})$ induced higher per cent haploid embryo formation in bread wheat than that preserved at $4{ }^{\circ} \mathrm{C}(60 \%$ $\mathrm{RH})$ and $-80^{\circ} \mathrm{C}(45 \% \mathrm{RH})$. These experimental results (in vitro and in vivo tests) hence confer that the best preservation regime for preservation of I. cylindrica pollen is $-20{ }^{\circ} \mathrm{C}$ $(65 \% \mathrm{RH})$. These results were in accordance with Towill (1985) who reported that many pollens store well at storage temperatures between -10 and $-35^{\circ} \mathrm{C}$.

From all the results obtained throughout the investigation, it is concluded that the best genotype for pollen preservation is Ic-Pbr, especially up to 15 days of preservation and best preservation regime for preservation of $I$. cylindrica pollen is $-20{ }^{\circ} \mathrm{C}(65 \% \mathrm{RH})$. By preserving the pollen of $I$. cylindrica at $-20^{\circ} \mathrm{C}(65 \% \mathrm{RH})$, it can be quite efficiently used for extending the period of $\mathrm{DH}$ production in wheat by at least one month or for undertaking $\mathrm{DH}$ breeding programmes at places where I. cylindrica is not growing naturally. For extending the $\mathrm{DH}$ breeding programmes by at least one month at places where I. cylindrica is available, best time to collect $I$. cylindrica pollen is the last days of its flowering under north-west Himalayan conditions. Whereas, for undertaking doubled haploidy breeding programmes in wheat at places where I. cylindrica is not growing naturally, best time to collect I. cylindrica pollen is initial days of its flowering.

\section{References}

Bajaj, Y.P.S. 1987. Cryopreservation of pollen and pollen embryos, and the establishment of pollen banks. Int. Review of Cytol. 107:397-420.

Barnabas, B. 1984. Freeze preservation of pollen. In: Proc. $5^{\text {th }}$ Symp. Internat. sur La Pollination. Versailles, France. INRA Publications 21:429-433.

Barnabas, B., Rajki, E. 1981. Fertility of deep-frozen maize (Zea mays L.) pollen. Ann. Bot. 48:861-864.

Chaudhary, H.K., Katoch, P.C., Mittal, R.K. 2005a. Practical Manual, Genetics and Plant Breeding. CSK Himachal Pradesh Agricultural University Printing Press. Palampur, Himachal Pradesh, India.

Chaudhary, H.K., Sethi, G.S., Singh, S., Pratap, A., Sharma, S. 2005b. Efficient haploid induction in wheat by using pollen of Imperata cylindrica. Plant Breeding 124:96-98.

Ganeshan, S., Rajasekharan, P.E., Shashikumar, S., Decruze, W. 2008 Cryopreservation of pollen. In: Reed, B.M. (ed.), Plant Cryopreservation: A Practical Guide. Springer. New York, USA. pp. 443-464.

Hanna, W.W., Towill, E.E. 1995. Long-term pollen storage. Plant Breeding Reviews 13:179-207.

Heslop-Harrison, J., Heslop-Harrison, Y., Shivanna, K.R. 1984. The evaluation of pollen quality and a further appraisal of the fluorochromatic (FCR) test procedure. Theor. Appl. Genet. 67:367-375.

Heslop-Harrison, J., Heslop-Harrison, Y. 1992. Germination of monocolpate angiosperm pollen: effects of inhibitory factors and the $\mathrm{Ca}^{2+}$-channel blocker, nifedipine. Ann. Bot. 69:395-403.

Hoekstra, F.A. 1995. Collecting pollen for genetic resources conservation. In: Guarino, L., Rao, V.R., Ried, R. (eds), Collecting Plant Genetic Diversity: Technical Guidelines. CAB International, Wallingford, UK. pp. 527-550.

Inagaki, M.N., Mujeeb-Kazi, A. 1994. Storage of maize pollen for use in haploid production of hexaploid wheat. Breeding Sci. 44:387-390. 
Inagaki, M.N., Mujeeb-Kazi, A. 1998. Production of polyhaploids of hexaploid wheat using stored pearl millet pollen. Euphytica 100:253-259.

Kubo, A. 1958. On the artificial pollen grain germination of Zea mays L. Botanical Magazine (Tokyo) 71:282285.

Kumar, A., Chowdhury, R.K., Dahiya, O.S. 1995. Pollen viability and stigma receptivity in relation to meteorological parameters in pearl millet. Seed Sci. and Technol. 23:147-156.

Mulugeta, D., Maxwell, B.D., Dyer, W.D. 1994. Kochia (Kochia scoparia) pollen dispersion, viability and germination. Weed Sci. 42:548-552.

Rangaswamy, R. 2006. Factorial experiments. In: A Text Book of Agricultural Statistics. New Age International (P) Ltd. Publishers. New Delhi, India. pp. 102-119.

Singh, R.J. 2003. Handling of plant chromosomes. In: Plant Cytogenetics. CRC Press LLC, Boca Raton, FL, USA. pp. 7-58.

Stanley, R.G., Linskens, H.F. 1974. Pollen: Biology, Biochemistry and Management. Springer. New York, USA.

Towill, L.E. 1985. Low temperature and freeze-/vacuum-drying preservation of pollen. In: Kartha, K.K. (ed.), Cryopreservation of Plant Cells and Organs. CRC Press. Boca Raton, FL, USA. pp. 171-198.

Towill, L.E. 2004. Pollen storage as a conservation tool In: Guerrant, E.O., Havens, K., Maunder, M. (eds), Ex Situ Plant Conservation: Supporting Species Survival in the Wild. Island Press, Washington DC, USA. pp. $180-188$.

Visser, T., De Vries, D.P., Welles, G.W.H., Scheurink, J.A.M. 1977. Hybrid tea rose pollen. I. Germination and storage. Euphytica 26:721-728.

Weatherhead, M.A., Grout, B.W.W., Henshaw, G.G. 1978. Advantages of storage of potato pollen in liquid nitrogen. Potato Res. 21:331-334.

\section{Electronic Supplementary Material (ESM)}

Electronic Supplementary Material (ESM) associated with this article can be found at the website of CRC at http://www.akademiai.com/content/120427/

Electronic Supplementary Table S1. Temperature $\left({ }^{\circ} \mathrm{C}\right)$ and relative humidity $(\%)$ on different days of Imperata cylindrica pollen collection (collection environments)

Electronic Supplementary Figure S1. Imperata cylindrica genotype having spike with brown anthers (Ic-Pbr)

Electronic Supplementary Figure S2. Imperata cylindrica genotype having spike with yellow anthers (Ic-Pye) 